Cahiers $d u$ MONDE RUSSE

\section{Cahiers du monde russe}

Russie - Empire russe - Union soviétique et États indépendants

$43 / 4 \mid 2002$

Intellectuels et intelligentsia

\title{
Sheila Fitzpatrick, Le stalinisme au quotidien
}

\section{Laurent Coumel}

\section{OpenEdition \\ Journals}

Édition électronique

URL : https://journals.openedition.org/monderusse/4035

DOI : 10.4000/monderusse. 4035

ISSN : $1777-5388$

Éditeur

Éditions de l'EHESS

Édition imprimée

Date de publication : 30 décembre 2002

Pagination : 736-739

ISBN : 2-7132-1796-2

ISSN : $1252-6576$

\section{Référence électronique}

Laurent Coumel, «Sheila Fitzpatrick, Le stalinisme au quotidien », Cahiers du monde russe [En ligne], 43/4 | 2002, mis en ligne le 17 juin 2009, consulté le 03 septembre 2022. URL : http://

journals.openedition.org/monderusse/4035; DOI : https://doi.org/10.4000/monderusse.4035

Ce document a été généré automatiquement le 3 septembre 2022.

Tous droits réservés 


\title{
Sheila Fitzpatrick, Le stalinisme au quotidien
}

\author{
Laurent Coumel
}

\section{RÉFÉRENCE}

Sheila FITZPATRICK, Le stalinisme au quotidien. La Russie soviétique dans les années 30. Paris, Flammarion, 2002, $415 \mathrm{p}$.

1 La traduction en français de cet ouvrage paru en 1999 aux États-Unis permettra à un large public de prendre connaissance des travaux récents d'une des historiennes de l'URSS les plus fécondes de sa génération. Après avoir étudié notamment l'enseignement et la mobilité sociale dans l'entre-deux-guerres, la « Révolution culturelle» (1928-1931) et dernièrement l'attitude des campagnes face aux transformations des années $1930^{1}$, Sheila Fitzpatrick revient sur cette décennie pour décrire et analyser « la vie ordinaire en des temps extraordinaires ", cette fois dans les villes russes. Pour mener à bien cette tâche immense, elle met à profit plus de trente années consacrées à l'histoire sociale de l'Union soviétique; une réflexion épistémologique accompagne en permanence le récit, illustrant le rapport étroit qui doit lier, ici plus qu'ailleurs, l'objet à la méthode. Ainsi l'omniprésence de l'État (entendu comme État-parti) l'a-t-elle poussée à adopter une définition inhabituelle du " quotidien»: celui-ci recoupe «l'ensemble des interactions quotidiennes impliquant l'État à un degré ou à un autre ", et exclut certains aspects qu'on pourrait s'attendre à voir développés ici (vie privée et loisirs). L'enchaînement des chapitres thématiques permet au lecteur de s'immerger par paliers dans cet univers déroutant, en découvrant successivement l'arrière-fond idéologique et politique, les difficultés matérielles, les modèles de réussite et les formes d'exclusion, les drames familiaux et, pour finir, les modes de surveillance et de répression - jusqu'à plonger dans l'irrationnel de la terreur de 1937-1938. La perception "d'en bas » du stalinisme met l'accent sur les individus, leurs «formes de comportement » et leurs «stratégies personnelles ». Quelques lignes ne suffisent pas pour résumer une réalité aussi complexe, dont l'étude mobilise une grande variété de sources : archives - y compris provinciales -, 
témoignages directs - écrits intimes et autobiographiques -, ou indirects (on notera ici le précieux apport du « Projet de Harvard " réalisé après la guerre auprès d'ex-citoyens soviétiques réfugiés aux États-Unis), presse et publications de l'époque, que complète un large horizon de références tirées de cinquante années de soviétologie et d'historiographie (les recherches dirigées par Sheila Fitzpatrick elle-même à l'université de Chicago fournissant une contribution majeure).

2 Le constat de départ, incontournable, est que la société russe des années 1930 est tout entière construite sur la pénurie, résultat d'une politique désastreuse - la collectivisation forcée des campagnes, la suppression du réseau de commerce privé, et la priorité absolue accordée à l'industrie lourde. En conséquence, le manque de produits alimentaires (cependant moins grave dans les villes que dans les campagnes), la crise du logement (liée à l'exode rural provoqué par les famines), les difficultés à se procurer certains objets usuels (chaussures, couteaux et autres ustensiles en métal) constituèrent des phénomènes douloureusement ressentis par la population, qui subissait alors les files d'attente et le rationnement (généralement considéré dans l'opinion comme un moindre mal), l'introduction du passeport intérieur (1932), mais aussi le discours officiel sur « l'avenir radieux », dont il ne faut pas négliger l'impact sur la faculté de résignation générale, ainsi que sur l'enthousiasme de certains activistes, des jeunes en particulier. La forte baisse du niveau de vie par rapport aux années de la NEP produisit deux effets à long terme sur le comportement du citoyen soviétique moyen: d'une part l'accès aux biens devint la motivation première de son existence, d'autre part le blat, "système de relations personnelles" (qu'on peut aussi traduire en français par "piston», mais il s'agit d'échanges d'égal à égal), et la recherche de protecteurs furent les moyens les plus répandus pour se procurer aliments, vêtements, appartement, soins médicaux, inscription à l'université, promotion, voiture, datcha, etc. Ainsi se mit en place un "État paternaliste » qui allait perdurer bien longtemps après la mort de Stalin - la formule «État-providence » employée en conclusion est peut-être plus discutable.

3 Les phénomènes d'ascension sociale sont eux aussi relus à la lumière de ce contexte de pénurie. En effet l'accès aux biens apparaît comme le moteur principal des aspirations de promotion, même si une autre dimension a pu jouer : le désir d'accéder à la culture, sinon à la gloire - dont témoigna l'engouement de la plupart des stakhanovistes pour les études supérieures, conformément à une sorte de marché implicite avec le régime. Devenus ingénieurs, enseignants, dirigeants locaux ou nationaux, les promus des années 1930 ont à la fois intériorisé l'idéal de progrès du régime (ils se considéraient comme l'avant-garde d'un mouvement qui ferait sortir l'ensemble du pays de "l'arriération »), et la hiérarchie des privilèges qui les séparait désormais du lot commun - car elle paraissait nécessaire à l'émancipation future de tous leurs concitoyens. Les récits fabuleux des exploits des héros soviétiques (aviateurs, explorateurs polaires) et la publicité pour de nouveaux délices tels que le ketchup et les crèmes glacées industrielles devaient faire patienter le reste de la population en attendant l'abondance promise.

4 Les années 1935-1936 semblent constituer une charnière, avec le tournant illustré dans la propagande par la célèbre formule prononcée par Stalin (et devenue un slogan courant, souvent tourné en dérision): «la vie est devenue meilleure, camarades; la vie est devenue plus joyeuse». On peut y voir à la fois une confirmation de la promesse du bonheur que ne devait pas manquer d'apporter la « construction socialiste », et un signe indiquant que les discriminations de classe et les purges allaient baisser d'intensité. Il est vrai que les privilégiés de l'époque, avant tout les hauts fonctionnaires et dirigeants du 
parti, mais aussi les stakhanovistes et certains membres de l'intelligentsia, goûtèrent alors un confort qui n'existait pas pendant la NEP (domestiques, maisons de repos, datchas, voitures étrangères), alors que le «mouvement des épouses" de dirigeants affichait un retour aux valeurs bourgeoises, inconcevable quelques années plus tôt. Les mis à l'écart de la société, les fameux lišency (« privés » de droits civiques), apprécièrent sans doute quant à eux la fin de la "guerre de classe " annoncée par la Constitution de 1936 - mais beaucoup continuèrent à souffrir des stigmates liés à leur passé, ou à les dissimuler dans l'angoisse d'être un jour "démasqués». Parallèlement, pour d'autres groupes comme les minorités nationales et les marginaux des grandes villes, s'annonçait " une sorte d'épuration sociale ». Surtout, les purges de 1937-1938 allaient refaire jouer le critère de l'origine sociale avec des conséquences funestes, et infliger à toute la population urbaine, et en premier lieu aux élites - mais la focalisation sur ce dernier groupe tient peut-être à leur sur-représentation dans les sources disponibles - un traumatisme sans précédent: "l'expérience sociétale de la terreur est non seulement celle des persécutés, mais aussi celle des persécutions [...]. Des gens qui n'avaient jamais volontairement dénoncé personne au cours des purges ont renoncé à défendre des amis cloués au pilori, ont cessé de fréquenter les familles des 'ennemis du peuple' et se sont retrouvés impliqués de mille façons dans le processus de la terreur. Un des principaux avantages des analyses fondées sur l'opposition entre 'eux' et 'nous' pour les citoyens soviétiques est de voiler cette réalité insupportable ; c'est une des principales raisons de la prudence dont devraient faire preuve les historiens à leur égard." Plutôt que de reconstituer les mécanismes explicatifs de la terreur- il est vrai que, vu depuis l'intelligentsia de l'époque, il n'y avait pas d'explication valable - Sheila Fitzpatrick rappelle dans quel contexte elle a été déclenchée, et comme elle a été difficile à contrôler, puis à stopper, tant la "culpabilité par association" n'en finissait pas de désigner de nouveaux suspects. Pourtant, une vérité non moins terrible émane des témoignages des contemporains : « la terreur n'était pas la terreur pour tout le monde ».

Pourquoi n'y eut-il pas davantage d'opposition au régime? Même si elle n'aborde pas de front cette question, Sheila Fitzpatrick apporte des éléments de réponse : la dépendance vitale qui relie tout citoyen à l'État distributeur de biens, la surveillance et la répression, l'absence de canaux d'expression et d'organisation collective expliquent la passivité générale de cette société. Cependant, le mécontentement était largement majoritaire dans l'opinion (même si l'hostilité était moins forte dans les villes que dans les campagnes), ce qu'illustrent les rapports du NKVD, les lettres anonymes envoyées aux dirigeants, mais aussi le timide débat public suscité par le régime lui-même à l'occasion de la Constitution et de la loi interdisant l'avortement en 1936.

Indirectement, l'ouvrage nous invite à une approche nouvelle de l'histoire politique de l'URSS des années 1930. La façon neutre dont Sheila Fitzpatrick rapporte certains discours de Stalin, en soulignant au passage leur "hypocrisie » ou leur " ambiguïté », relève d'un parti pris de neutralité qui peut dérouter le lecteur - même s'il n'est pas dénué d'ironie. Une telle distance, qui évite à la fois la fascination et l'horreur que peut inspirer le personnage, s'avère des plus efficaces pour percevoir la mentalité du citoyen soviétique moyen, pour qui Stalin est justement ce monstre froid, cet oracle qui lance des messages - les « signaux » qu'il faut interpréter - et peut d'un simple mot changer le cours de l'existence, tel un père de famille. Surtout, les pages concernant la vie quotidienne des dirigeants (leurs privilèges et l'idée qu'ils s'en faisaient, les rapports avec leurs « clients ", leur conception de leur propre rôle) s'avèrent fondamentales pour appréhender le fonctionnement des cercles du pouvoir. Pour faire le bilan de cette 
«exploration du quotidien et de l'extraordinaire dans la Russie de Stalin et de l'interaction de l'un et de l'autre ", il faut revenir sur le sentiment dominant de l'opinion de l'époque: celui de ne pas vivre "une vie normale». Les pénuries et le manque de confort, un système paternaliste d'accès aux biens, une idéologie devenue religion, une ligne politique imprévisible, des lendemains qui chantent mais restent incertains, l'omniprésence des ennemis et l'impossibilité de leur rachat aux yeux du régime, l'arbitraire de la répression policière, tout cela pesa et transforma le caractère de l'homme et de la femme soviétiques des années 1930, qui trouvèrent diverses réponses appropriées: sentiment de ne pas participer au pire (voir l'analyse critique de l'opposition entre "eux" et "nous", supra), repli sur la sphère familiale, formes anonymes de subversion (chansons, histoires drôles), pétitions adressées aux protecteurs éventuels, stratégies de survie, évasion vers d'autres mondes... Si l'on peut regretter l'absence du lieu de travail, ou encore des formes de culture de masse comme le cinéma, c'est bien la civilisation stalinienne (et, partant, comme elle le dit elle-même, soviétique) que Sheila Fitzpatrick embrasse dans son ensemble, mettant au jour les traits durables du système, plutôt que des événements politiques isolés. Ce faisant elle offre, après quelques études pionnières comme celle de Stephen Kotkin sur Magnitogorsk, une première synthèse sur la question. Une telle approche semble pouvoir concilier les deux directions habituellement opposées de la recherche sur le stalinisme; notons que, si elle n'hésite pas à souligner les points de comparaison avec le régime nazi, Sheila Fitzpatrick emploie très peu le terme « totalitaire ».

\section{NOTES}

1. Stalin's peasants: resistance and survival in the Russian village after collectivization, New York, 1994 - qui a déjà été traduit en russe, mais pas en français malheureusement. 